\title{
Quand l'indication des inlay-cores métalliques
devient incontournable
}

\section{When metallic post and core restoration is inevitable}

\section{MOTS-CLEFS : \\ - Inlay-core \\ reconstitution corono- radiculaire métallique, tenon radiculaire, dent dépulpée}

\section{KEYWORDS:}

- Cast post and core, metallic post and core, restoration,

root post, devitalized tooth

\section{Résumé}

En matière de restauration prothétique, on s'oriente de plus en plus vers des reconstitutions sans métal, plus esthétiques et aux propriétés mécaniques améliorées. Cependant, les reconstitutions coronoradiculaires métalliques indirectes restent irremplaçables dans certains cas.

En plus du recul clinique acquis de cette technique, plusieurs avantages, tels que les propriétés mécaniques, et la simplicité de réalisation au laboratoire, font que les inlaycores métalliques sont encore fréquemment utilisés.

Quelles sont les indications de ces reconstitutions et les facteurs de décision et comment ont-elles évolué pour rester toujours d'actualité ?

\begin{abstract}
In terms of prosthetic restoration, dentists are moving more and more towards freemetal restorations, more aesthetic with equal mechanical properties. However, in some cases, using a metallic post and core for the coronal-radicular reconstruction remains irreplaceable.

In addition to the acquired clinical experience, this technique presents several benefits, such as mechanical properties, and ease of acheivement in the laboratory. All these reasons uphold the continuous use of the metallic post and core that frequently beneath fixed partial dentures.

When should we use this kind of restoration? What are the decision parameters leading to this choice? And what makes them updated?
\end{abstract}

AOS 2014;269:16-21 DOI: $10.1051 / \mathrm{aos} / 2014304$ (C) EDP Sciences 2014

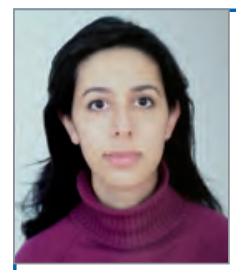

- Layla ASSILA, Chirurgien-dentiste, spécialiste et PhDc au service de prothèse conjointe. Faculté de médecine dentaire, Rabat, Maroc.

Loubna EL FIGUIGUI, Chirurgien-dentiste, spécialiste et PhDc au service de prothèse conjointe. Faculté de médecine dentaire, Rabat, Maroc.

Hicham SOUALHI, Professeur assistant au service de prothèse conjointe. Chirurgien-dentiste, résidente et $\mathrm{PhDc}$ au service de prothèse conjointe. Faculté de médecine dentaire, Rabat, Maroc.

Amal EL YAMANI, Professeur d'enseignement supérieur, chef de service de prothèse conjointe. Faculté de médecine dentaire, Rabat, Maroc. 


\section{INTRODUCTION}

Dans notre pratique quotidienne, nous sommes souvent confrontés à des cas présentant des destructions coronaires importantes. Ce sont des situations qui nécessitent impérativement de réaliser une reconstitution corono-radiculaire (RCR) avant toute restauration prothétique.

Lors du choix d'un plan de traitement optimal, le médecin-dentiste est parfois influencé par l'évolution des techniques et des matériaux de restauration en faveur d'une restauration esthétique en réponse aux exigences des patients.

Mis à part ces facteurs extrinsèques influençants, quels sont les critères de décision en faveur d'un inlay-core métallique ? Répondent-ils aux exigences prothétiques actuelles, biologiques, fonctionnelles et esthétiques?

\section{POURQUOI UNE RCR ?}

Une reconstitution corono-radiculaire est une restauration qui intéresse à la fois la partie coronaire et la partie radiculaire de la dent.

Elle concerne toujours une reconstitution complexe qui, pour assurer sa rétention, s'adresse à des ancrages radiculaires [1-3].

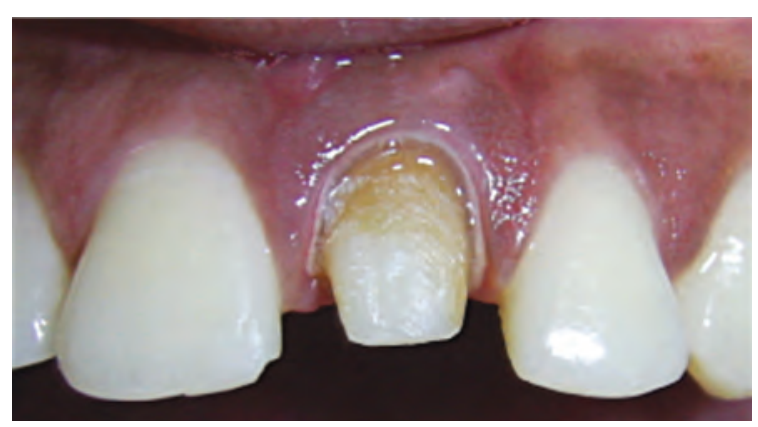

$\triangle$ Fig. 1 :

Reconstitution corono-radiculaire foulée : tenon en fibres + composite.
Une RCR permet :

$\checkmark$ de restaurer la perte de substance due à un traumatisme ou une lésion carieuse et leurs thérapeutiques ;

$\checkmark$ de préserver les tissus dentaires résiduels ;

Id'assurer l'étanchéité de l'obturation canalaire ;

$\checkmark$ d'assurer la rétention de la pièce prothétique susjacente ;

$\checkmark$ une répartition équilibrée des contraintes subies par la restauration prothétique et transmises aux tissus, coronaire et radiculaire, restants ;

$\checkmark$ de contribuer à la restauration de l'aspect esthétique de la restauration $[1,4]$.

\section{QUELS SONT LES TYPES DE RCR ? [1, 3, 5, 6]}

On distingue de façon conventionnelle :

$\checkmark$ les RCR directes : utilisant des matériaux insérés en phase plastique, soutenues ou non par un tuteur (fig. 1);

$\checkmark$ les RCR indirectes : réalisées au laboratoire. Elles sont métalliques (alliages précieux ou non précieux) ou en céramique (fig. 2).

Le choix entre l'une ou l'autre de ces deux catégories est pour le plus souvent basé sur un critère qui semble être le plus important : la quantité de substance coronaire résiduelle. Cependant, plusieurs autres critères décisionnels doivent être pris en considération, et peuvent orienter notre choix vers l'utilisation d'un inlay-core métallique, ils seront illustrés par des situations cliniques.

\section{CRITÈRES EN FAVEUR D'UNE RCR COULÉE}

La réalisation d'une RCR foulée ou coulée est un acte quotidien dont le choix est souvent basé sur les exigences esthétiques du patient ou le nombre de
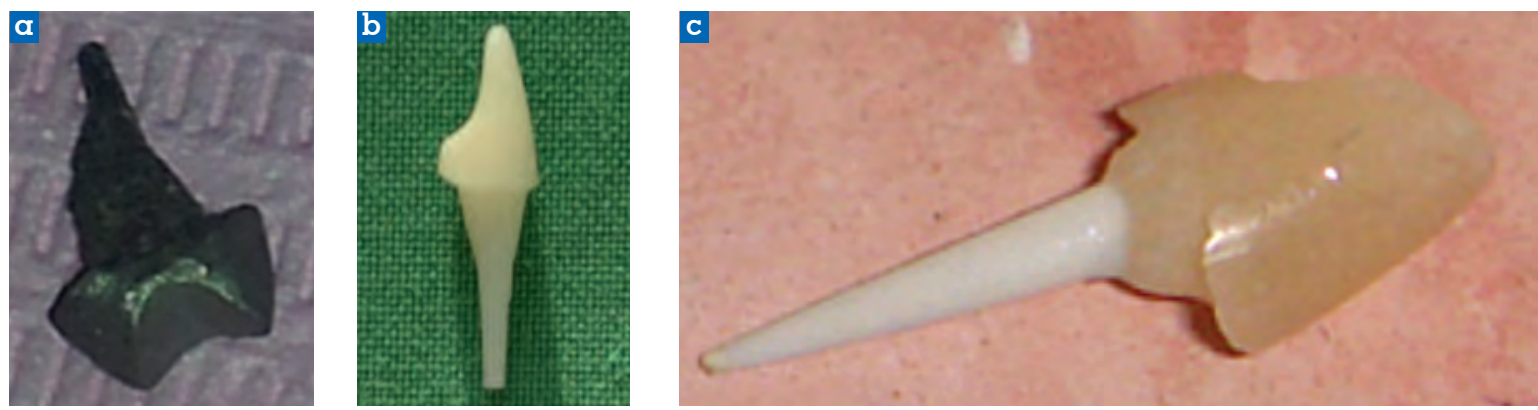

$\triangle$ Fig. 2 : Reconstitution corono-radiculaire coulée.

a. Inlay-core métallique.

b. Inlay-core tout céramique.

c. Tenon en céramique et faux moignon en composite de laboratoire. 


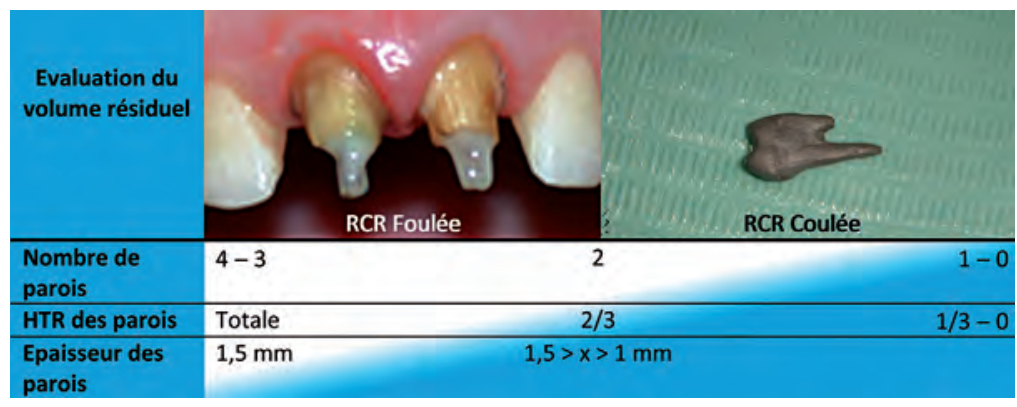

4 Tableau I :

Choix entre RCR foulée et coulée selon le nombre des parois résiduelles, leur hauteur et leur épaisseur

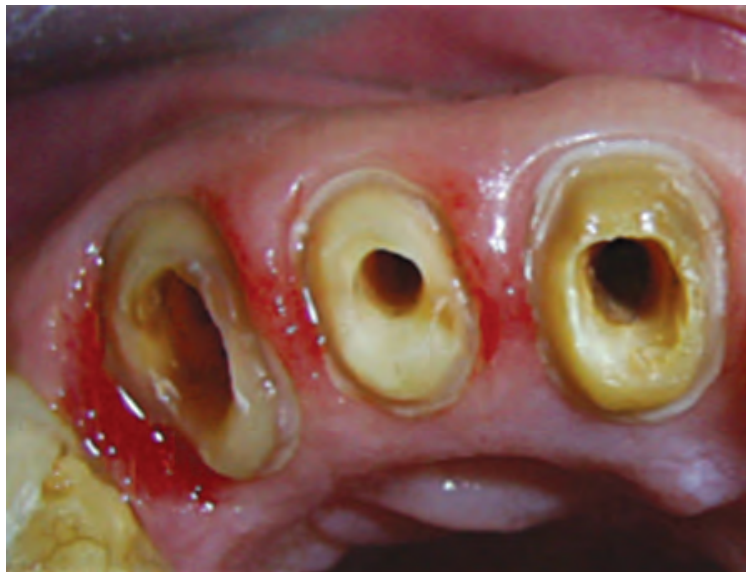

Fig. 3 :

La canine présente un délabrement plus important que celui de la centrale, avec une limite cervicale intrasulculaire, ce qui impose le choix d'un inlay-core métallique sur cette dent.

parois résiduelles après préparation périphérique. Ce ne sont pas les seuls critères de décision dont il faut prendre compte, notamment face à des situations intermédiaires ( 2 parois restantes). Après une analyse complète du cas, l'inlay-core métallique peut s'avérer l'unique choix thérapeutique idéal qui permettra de réaliser une restauration prothétique étanche, rétentive et offrant une bonne intégration dans le contexte occluso-parodonto-prothétique.

\section{VOLUME CORONAIRE RÉSIDUEL $[1,3,6,7]$}

Il est évalué sommairement par le nombre de parois restantes après une préparation corono-périphérique adéquate. Est considérée comme paroi, celle ayant une épaisseur supérieure à $1,5 \mathrm{~mm}$ et une hauteur supérieure à la moitié de la hauteur coronaire totale (tableau I).

Si ce critère peut s'avérer suffisant dans les cas extrêmes ( 0 paroi ou 4 parois restantes), une configuration clinique intermédiaire ( 2 parois restantes) impose la prise en considération d'autres facteurs (anatomique, fonctionnel, technologique et contextuel) dans la prise de décision thérapeutique.

\section{SITUATION DE LA LIMITE CERVICALE $[1,5,6,8,9]$}

Face à un délabrement coronaire important, la préparation périphérique impose souvent des limites cervicales en juxta-gingival ou intra-sulculaire. Dans ces cas-là, l'utilisation d'une RCR foulée se trouve contre-indiquée pour deux raisons :

I'emploi du composite pour le collage impose une mise à l'abri des fluides buccaux ;

$\checkmark$ l'étanchéité cervicale n'est garantie qu'en présence d'au moins $2 \mathrm{~mm}$ de tissus dentaires entre le niveau gingival et la limite de la RCR foulée.

Pour cela, l'existence d'un limite cervicale juxta- ou infra-gingivale ou à moins de $2 \mathrm{~mm}$ de la limite dento-prothétique (fig. 3) impose l'utilisation d'un inlay-core métallique coulé à la place d'une RCR foulée.

\section{SECTEUR ANTÉRIEUR $[1,2,6]$}

Au niveau du secteur antérieur, les dents présentent un volume coronaire faible (en comparaison avec les molaires) notamment en épaisseur vestibulo-palatine (surtout les dents inférieures) dont la restauration au composite ou à la céramique est contre-indiquée car seul le métal présente une bonne résistance mécanique sous de faibles épaisseurs (fig. 4).

De plus, ces dents sont soumises à des forces de flexion qui, associées à une préparation coronaire plus haute que large, vont créer un bras de levier important sur l'élément prothétique sus-jacent soumis à des forces tangentielles.

Il est alors recommandé d'utiliser un matériau ayant une bonne résistance mécanique sous les forces de flexion.

Pour une RCR foulée, les tenons en fibres présentent certes une bonne résistance à la flexion (1 400 à $1800 \mathrm{MPa}$ ). Cependant, le composite présente une résistance insuffisante au cisaillement et à la flexion (60 à $150 \mathrm{MPa}$ ), contrairement aux alliages utilisés pour les RCR coulées qui présentent une meilleure résistance mécanique. 

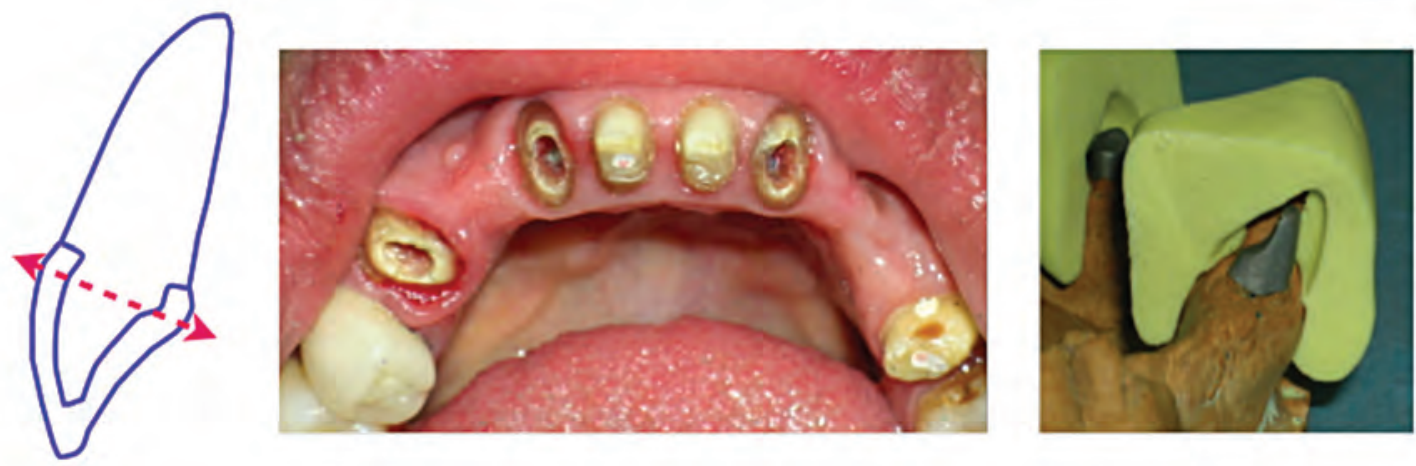

$\triangle$ Fig. 4 :

Une épaisseur vestibulo-palatine faible résiduelle à la préparation périphérique au niveau du secteur antérieur, notamment au niveau inférieur. Quand l'indication d'une RCR est posée, les alliages non précieux présentent une meilleure résistance sous de faibles épaisseurs et sous des forces tangentielles de flexion.

$\nabla$ Tableau II :

Comportement mécanique des tissus dentaires, des alliages utilisés en prothèse fixée et des tenons en fibres.

\begin{tabular}{|c|c|c|c|c|}
\hline \multicolumn{2}{|c|}{ Matériaux } & Dureté (Vickers) & Module d'élasticité (GPa) & Résistance à la flexion (MPa) \\
\hline \multicolumn{2}{|c|}{ Email } & $240-440$ & 84 & - \\
\hline \multicolumn{2}{|c|}{ Dentine } & $50-87$ & 18 & - \\
\hline \multicolumn{2}{|c|}{ Or } & $40-230$ & $90-117$ & $286-790$ \\
\hline \multirow{2}{*}{$\begin{array}{l}\text { Alliages non } \\
\text { précieux }\end{array}$} & $\mathrm{Ni}-\mathrm{Cr}$ & $210-380$ & $150-210$ & \multirow{2}{*}{400 - 800 limite de rupture } \\
\hline & $\mathrm{Co}-\mathrm{Cr}$ & $330-465$ & $145-220$ & \\
\hline \multicolumn{2}{|c|}{ Tenons en fibres de quartz } & & $18-50$ & $1400-1800$ \\
\hline
\end{tabular}

ANATOMIE RADICULAIRE [1, 3, 6, 10, 11$]$

Le tenon d'une RCR a pour rôle d'offrir un ancrage canalaire à la restauration et d'en majorer la rétention. La corrélation entre ces principes mécaniques et quelques particularités morphologiques (racine grêle ou aplatie, courbure radiculaire...), tout en respectant les critères cliniques d'une préparation radiculaire, rend incontournable l'utilisation de tenons anatomiques, moins mutilants et plus rétentifs, qui ne sont concevables qu'en RCR coulée.

La restauration d'une dent présentant une racine fragile oriente le choix vers une RCR alliant le respect de l'économie tissulaire et de l'anatomie canalaire, aux propriétés mécaniques adaptées. Actuellement les tenons en fibres constituent l'indication majeure (grâce à leurs propriétés proches de celles des tissus dentaires). Cependant, en rapport avec l'étendue du délabrement, l'anatomie radiculaire, et le contexte général de la situation clinique, lindication de RCR indirecte est préconisée. Ainsi l'utilisation des alliages précieux, aux propriétés mécaniques compatibles avec celles des tissus dentaires, constitue un meilleur compromis puisqu'ils permettent d'allier les impératifs précités et le respect de la fragilité intracanalaire (tableau II).

\section{CONTEXTE GLOBAL}

Une restauration corono-radiculaire doit être placée au sein d'un plan de traitement global prenant en compte le contexte fonctionnel, parodontal et prothétique.

\section{Contexte fonctionnel}

Les dents qui subissent des contraintes non axiales, fonctionnelles ou parafonctionnelles, nécessitent une $\mathrm{RCR}$ réalisée par un matériau qui résiste à ce genre de forces $[1,5]$.

\section{Contexte parodontal}

Face à un parodonte réduit, la restauration prothétique, et ce dès les premières étapes du traitement, doit 

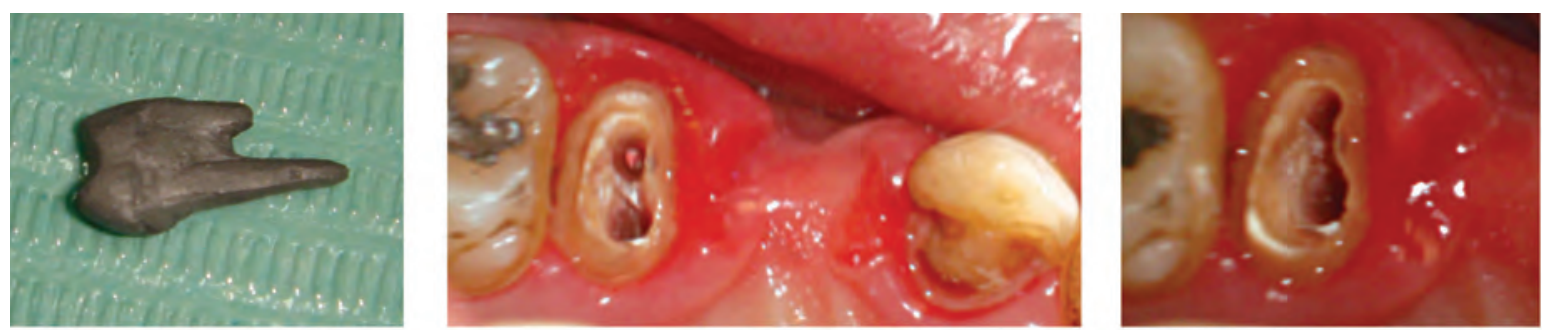

$\triangle$ Fig. 5 :

Les inlay-cores métalliques respectent mieux l'anatomie canalaire à morphologie particulière (par exemple la forme en 8 des prémolaires) et offrent un meilleur rapport mutilation-rétention.

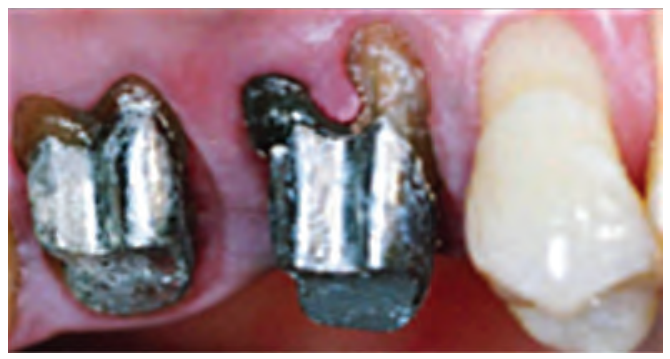

$\triangle$ Fig. 6 :

Face à un parodonte réduit, les inlays-cores métalliques permettent d'obtenir des formes de restauration à morphologie adaptée.

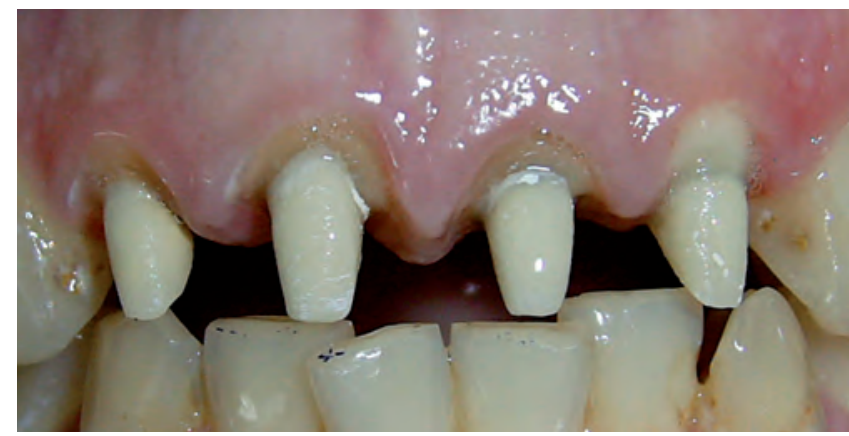

Fig. 7 :

Parallélisme des piliers rattrapés par des inlay-cores.

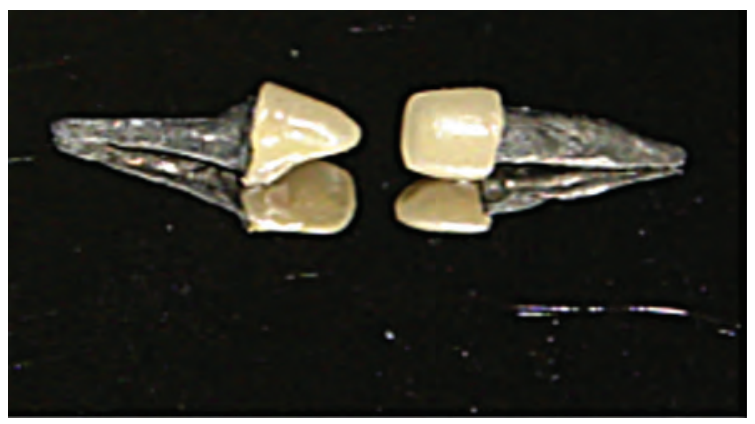

$\triangle$ Fig. 8 :

Les inlays-cores céramisés au niveau des incisives centrales permettent d'éviter l'aspect grisâtre inesthétique de l'inlay-core.

répondre aux exigences d'une hygiène correcte par la réalisation de reconstitutions à morphologie adaptée. Ces formes de préparations périphériques, suivant les contours sinueux causés par les pertes d'attaches, sont facilement obtenues grâce à l'utilisation des inlay-cores métalliques [12] (fig. 5 et 6).

\section{Contexte prothétique}

Une réhabilitation prothétique utilisant une prothèse partielle amovible supportée par des couronnes fraisées exige le choix de couronnes céramo-métalliques.

Face à une prothèse plurale, l'obtention d'un parallélisme entre les piliers n'est pas toujours chose facile [13].

Devant ces différentes situations particulières, quand

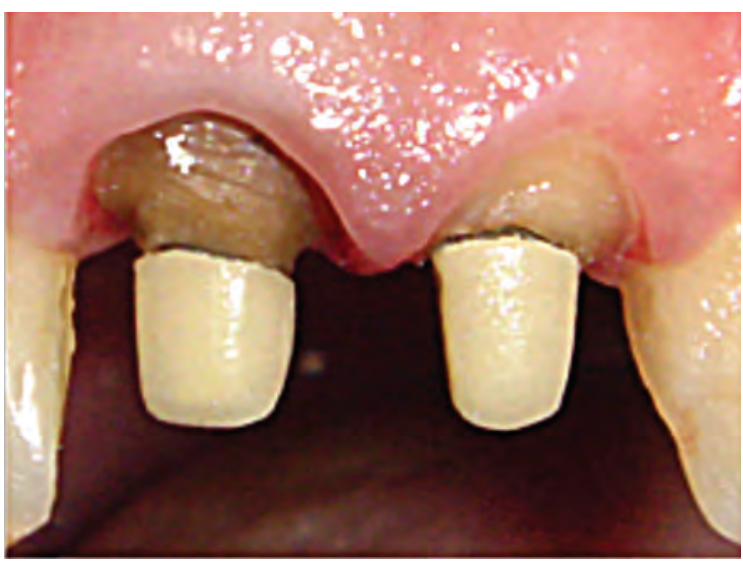

une reconstitution corono-radiculaire est incontournable, l'utilisation d'un inlay-core métallique (contrairement à une RCR foulée) permet :

$\checkmark$ de résister aux forces non axiales ;

$\checkmark$ d'utiliser des couronnes céramo-métalliques à morphologie adaptée (parodonte réduit ou PAP) ;

$\checkmark$ d'obtenir le parallélisme des piliers (fig. 7).

\section{INLAY-CORE ET ESTHÉTIQUE [14]}

Devant l'utilisation de plus en plus étendue des couronnes céramo-céramiques, l'inlay-core métallique représente une contrainte esthétique. Cependant, quand son indication est incontournable (paramètres 
cliniques précités), l'alternative se présente sous forme d'un inlay-core métallique céramisé, ou inlay-core en or (fig. 8).

\section{INLAY-CORE ET BIOCOMPATIBILITÉ}

Les alliages dentaires les plus concernés par la biocompatibilité et les phénomènes galvaniques sont, pour l'essentiel, les non précieux qui peuvent entraîner chez certains patients des réactions d'hypersensibilité. En effet, pour les alliages à haute teneur en or, aucune réaction de la muqueuse buccale à leur égard n'a été mise en évidence au cours d'une étude in vivo [15].

Actuellement, certains alliages $\mathrm{Ni}-\mathrm{Cr}$ sont commercialisés avec des certificats de biocompatibilité [16].

\section{CONCLUSION}

La réalisation d'une RCR foulée ou coulée est un acte quotidien dont le choix reste d'une grande difficulté et auquel est confronté chaque praticien [17].

Ce choix est souvent basé sur les exigences esthétiques du patient ou le nombre de parois résiduelles après préparation périphérique. Ce ne sont pas les seuls critères de décision dont il faut prendre compte, notamment face à des situations intermédiaires (deux parois restantes). Après une analyse complète du cas, l'inlay-core métallique reste une alternative toujours d'actualité dans l'ère de la dentisterie adhésive et peut même parfois s'avérer l'unique choix thérapeutique idéal qui permettra de réaliser une restauration prothétique étanche, rétentive et offrant une bonne intégration dans le contexte occluso-parodonto-prothétique.

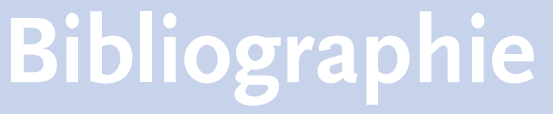

[1] Laplanche O, Leforestier E, Medioni E, Bolla M. Les reconstitutions corono-radiculaires : principes généraux et critères de décision. Stratégie prothétique 2008;8:255-268.

[2] Etienne O, Descamp F, Toledano C. Apport des reconstitutions collées en dentisterie esthétique. L'information Dentaire 2009;6:264270.

[3] Geoffrion J. Bartala M. Économie tissulaire et préparations des ancrages coronoradiculaires. Cah Prothèse 2003;124: 63-70.

[4] Bolla M, Bennani V. La reconstitution corono-radiculaire pré-prothétique des dents dépulpées. Paris: Éditions $C d P$, Collection Guide Clinique, 1999.

[5] Seguela V, Soenen A, Laviole O, Bartala M. Reconstitution corono-radiculaire et système tout-céramique. Cah Prothèse 2011;155:15-27.
[6] Laviole O, Bartala M. Restaurations coronaires pré-prothétiques à ancrage radiculaire : clinique et laboratoire. $E M C$ 2008;23-250-A-10.

[7] Goodacre CJ, Spolnik KJ. The prosthodontic management of endodontically treated teeth: a literature review. Part Tooth preparation considerations. J Prosthodont 1995;4(2):122-8.

[8] Koubi S, Weissrock G, Tassery H, Brouillet JL. Reconstitutions corono-radiculaires collées fibrées, qu'en est-il ? ID Inf Dent 2008;90(8):1382-1392.

[9] Lefevre X, Bolla M, Leforestier E, Bellet $M$. Influence de la nature du tenon radiculaire sur la répartition des contraintes au sein d'une reconstitution prothétique. Analyse par méthode des éléments finis. J Biomat Dent 2000;XV:117-128.

[10] Nergiz I, Schmage P, Ozcan M, Platzer U. Effect of length and diameter of tapered post on the retention. J Oral Rehab 2002;29(1):28-34.
[11] Dejou J, Laborde G. Le tenon radiculaire est-il indispensable, utile ou dangereux ? Cah Prothèse 2001;116:31-41.

[12] Borghetti A, Monnet-Corti V, Azzi R. Chirurgie plastique parodontale. Paris : Éditions CdP, Collection JPIO 2000:374-383.

[13] Laredo F. Reconstitutions des dents dépulpées support de prothèse fixée, à propos des reconstitutions prothétiques. Le Chirurgien Dentiste de France 2002;1092:27-35.

[14] Descamp F, Etienne O. Inlay-core, une technique encore d'actualité. Cah Proth 2010;150:59-68.

[15] Moulin P, Soffer E, Doukhan JY. Alliages précieux en odontologie. EMC 2002;23-065-P-10.

[16] Wataha JC. Alloys for prosthodontic restorations. J Prosthet Dent 2002;87(4):351-63.

[17] Borg-Real C, Bolla M, Leforestier E, Laplanche O, Muller-Bolla M. Choix d'un type de reconstitution corono-radiculaire. Revue systématique de la littérature. Cah Proth 2008;142:59-70. 